http://jmscr.igmpublication.org/home/ ISSN (e)-2347-176x ISSN (p) 2455-0450 crossref DOI: https://dx.doi.org/10.18535/jmscr/v8i11.57

\title{
The Effects of Zinc on Shortening the Duration of the Covid-19 Symptoms
}

Authors

\author{
Hassan Darwish, $\mathrm{PhD}^{\mathbf{1}}$, Lorna Schumann, $\mathrm{PhD}^{2}$, Jesse Hernandez ${ }^{3}$ \\ ${ }^{1}$ Berkeley Diabetes \&Botanical Research Institute, Laguna Beach, California, USA \\ ${ }^{2}$ American Academy of Nurse Practitioner Certification Board, Austin, TX, USA \\ Washington State University, Spokane, WA., USA \\ ${ }^{3}$ Iso-Tex Diagnostics Radiopharmaceutical, Friendswood, Texas, USA
}

\begin{abstract}
Background: The emergence and spread of Coronavirus Disease 2019 (COVID-19) brought about a public health crisis threatening the globe. Severe acute respiratory syndrome coronavirus 2 (SARS-CoV2), epitomizes society's greatest health problem and cause of death. Until a vaccine is developed, controlling the spread and supportive medications are essential. Zinc $(\mathrm{Zn})$ is one of the supportive treatments that have significant and implicit antiviral characteristics that are appreciated in the treatment of the common cold and enhancing the immune system.
\end{abstract}

Objectives: To evaluate the connection between daily dosage of zinc tablets/lozenges and the duration of colds in patients.

Methods: Ovid MEDLINE was examined utilizing the free search terminologies "zinc and lozenges" that produced over 60 research articles. Findings from the zinc trials were analyzed using, P-values together with the Fisher method and combining the survey outcomes through the RevMan @ program.

Results: High-dose zinc acetate (greater than $75 \mathrm{mg}$ ) tests provide a decline in the duration of colds, and there is "heterogeneity" observed between the tests.

Conclusions: Zinc lozenges can potentially reduce the duration of symptoms of the coronavirus when consumed within a day on the onset.

Keywords: Covid-19, Zinc (Zn), SARS-CoV-2.

\section{Introduction}

The years 2019-2020 will be remembered for the global pandemic of COVID-19 brought about by SARS-CoV-2. The illness was first recognized in "Wuhan, Hubei Province, China", in 2019. Due to the viruses' high contagiousness the disease has spread worldwide and compelled the World Health Organization (WHO) to declare the condition as a pandemic on March $11^{\text {th }}, 2020$, when the total global cases surpassed 500,000. By November $1^{\text {st }}, 2020$, the number of cases was 46,722, 172 with1, 204844 deaths.

COVID-19 epitomizes the greatest severe health problem for the world in the contemporary history of humans. On the March 6 $6^{\text {th }}, 2020$ (WHO) reported the crude mortality ratio as about 
3.5 which has decreased to $2.7 \%$ on July $12^{\text {th }}, 2020$ (Coronavirus Update (Live): 13,855,229 Cases and 589,947 Deaths from COVID-19 Virus Pandemic - Worldometer, 2020).

In contrast, these ratios differ according to nations, the age of the patients, and the presence of co-morbidities. Most patients who have succumbed to the disease are those aged 60 and above plus those with pre-existing health challenges, encompassing diabetes, cardiovascular diseases or conditions that affect the immune system.

SARS-CoV-2 is spread by inhalation or contact with infected droplets from one person to another, viral loads of symptomatic and asymptomatic carriers of the virus are similar. Like "flu and rhinovirus", the spread of SARS-CoV-2 is perceived to happen via respiratory droplets from sneezing and coughing (Cascella, 2020). The transfer of the virusoccurs when individuals are in close contact, defined as closer than 6 feet or 2 meters for more than 10 minutes. The disease can also be spread when one comes into contact with a contaminated surface, and then touches their nose, mouth and eye. Currently, treatment mechanisms to manage COVID-19 are being tested, but the only effective preventative measures for decreasing community spread is social distancing, self-isolation and wearing masks. However, some systematic reviews propose zinc tablet/lozenges likely decrease the duration of symptoms of the coronavirus.

Coronavirus represents the subfamily "Orthocoronavirinae, within the family realm Riboviria, Coronaviridae, and the order of Nidovirales" (Mousavizadeh \& Ghasemi, 2020). The viruses are enclosed with a definite "sense single-stranded ribonucleic acid genome and a nucleocapsid of helical symmetry" (Turner, 2016). The Wuhan strain was established as a new strain of Betacoronavirus from category $2 \mathrm{~B}$, with nearly $70 \%$ genomic comparation to the SARS-CoV (Johnstone et al., 2015). The virus originated in bats and was transmitted to humans by an unknown intermediary animal.
This review focuses on the use of Zinc tablets/lozenges to decrease the duration of COVID-19 and improve the immune system, most specifically the $\mathrm{T}$ cells. Zinc $\left(\mathrm{Zn}^{2+}\right)$ works in the overall improvement, variation, and operation of immune cells (Cascella, 2020).

Zinc is involved in different cellular procedures and presents several explicit and implicit antiviral characteristics. It has been shown that zinc deficiency is linked to decreased antibody production, reduced cytokine production by monocytes, the innate immune system's function, and the "chemotaxis and the oxidative burst of neutrophil granulocytes" (Johnstone et al., 2015).

Therefore, zinc has several influences on the immune structure, and its deficiency augments the susceptibility to diseases. In low resource nations, there is significant data that supports zinc supplementation to decrease the danger of contracting of common cold and pneumonia (Turner, 2016 5). However, these research data cannot be generalized to the developed nations such as France and the UK, where "multivitaminmineral tablets" consisting of about $18 \mathrm{mg}$ per day of zinc had no impact on the frequency of respiratory diseases in the elderly (Kumar et al., 2020). Interests in zinc lozenges for conventional cold therapy began from the opportune event of a cold in young woman with leukemia. When she liquefieda medicinalzinc lozenge in her mouth rather than consuming it the cold symptoms disappeared (Johnstone et al., 2015). Some zinc versus placebo controlled trials have conflicting findings.

The RNA synthesis of the virus is catalyzed by an RNA-dependent RNA polymerase ( $R d R p)$, which is the core enzyme of the multiprotein replication and transcription complex (RTC). Using an activity assay for RTCs isolated from cells infected with SARS-CoV, eliminating the need for PT to transport $\mathrm{Zn}^{2+}$ across the plasma membrane, it shows that $\mathrm{Zn}^{2+}$ efficiently inhibits the RNAsynthesizing activity of the RTCs for the virus. Enzymatic studies using recombinant RdRps (SARS-CoV) purified from E. coli subsequently 
revealed that $\mathrm{Zn}^{2+}$ directly inhibited the in vitro activity of the virus polymerases. More specifically, $\mathrm{Zn}^{2+}$ was found to block the initiation step of RNA synthesis, whereas in the case of the SARS-CoV RdRp elongation was inhibited and template binding reduced. By chelating $\mathrm{Zn} 2+$ with MgEDTA, the inhibitory effect of the divalent cation could be reversed, which provides a novel experimental tool for in vitro studies of the molecular details of virus replication and transcription.(te Velthuis et al., 2020).

\section{Methodology}

This systematic review study examined the available data of the pertinent literature relating to the COVID-19 and the use of Zinc, aiming to provide clinical evidence on Zinc tablets/lozenges intake and the duration of colds symptoms due to Covid-19 infections in patients. To achieve this goal a preliminary search was done on July $2^{\text {rd }}$, 2020 using Ovid MEDLINE to find out studies and scientific information that evaluate the ability of curative impacts of zinc on common cold diseases. All the studies which are published in English from 2015 to 2020 were included in the search. According to the status of observation, more than 80 studies related to Zinc tablets/ lozenges were found. To make the results more accurate: the following keywords were used: Covid-19, Zinc (Zn), SARS-CoV-2, and common cold. By using these keywords, the number of studies was reduced to less than 65. For the purpose of quality and specificity of the search the studies were excluded if they were opinion, review, text studies or systematic review. To avoid any factors that can affect the quality of the search and to increase its efficiency we filtered the studies that we obtained using PubMed search filter, Cochrane databases search filter and EMBASE search filter. As a result of using inclusion and exclusion criteria and other methods of filtering the number of studies was reduced to 15. These 15 studies were screened by examining the abstract, method and the results and finally the best 13 were selected based on the quality of the method and the results that have been found. All the selected studies evaluated the connection between daily dosage of zinc tablets/lozenges and the duration of colds in patients.

To make sure that their methods were high quality, the researchers did appraise them using the JBI Critical appraisal tools. The major type of checklist that is used is an experimental study checklist. By using this critical appraisal tool, the researchers were able to find the strengths and weaknesses of each study and concluded that the selected studies are at high quality to be used in any systematic review based on the JBI critical appraisal tools and inclusion and exclusion criteria. The process of identifying the studies is summarized in Figure 1.This methodical appraisal was limited to tests evaluating curative impacts of zinc on common cold diseases. Relieving pain without dealing with the cause was needed since experimentally having a clear decisive relevance to typical cold. The aggregate regular dose of rudimentary zinc from the tablets was computed as a product of the zinc dosage per tablet, and the designed number of tablets per day. 
Table 1. Effect of Zinc Lozenges on Common Cold Episodes of Natural Origin

\begin{tabular}{|c|c|c|c|c|c|c|}
\hline Study [Ref.] & No. of Participants & $\begin{array}{c}\text { Zn Dose } \\
\text { Per Day }(\mathrm{mg})^{\mathrm{a}}\end{array}$ & $\begin{array}{c}\text { Average Duration of Colds (Days) } \\
\text { Zn }{ }^{b} \text { Placebo }\end{array}$ & The Effect of $\mathbf{Z n}$ & $\underset{(1-T a i l)}{P^{b}}$ & $-2 \times \ln (\mathrm{P})$ \\
\hline Eby et al.1984 [7] & 65 & 207 & $3.9 / 10.8^{c}$ & $-64 \%{ }^{c}$ & $0.0005^{c}$ & 15.2 \\
\hline Smith et al.1989 [19] & 110 & 207 & $\begin{array}{l}5.9 / 6.3^{\mathrm{d}} \\
5.5 / 6.9^{\mathrm{d}}\end{array}$ & $-22 \%{ }^{d}$ & $\begin{array}{l}0.5^{\mathrm{d}} \\
0.01^{\mathrm{d}}\end{array}$ & 1.4 \\
\hline Godfrey et al. 1992 [20] & 73 & 192 & $4.9 / 6.1$ & $-21 \%$ & 0.024 & 7.4 \\
\hline Prasad et al. 2008 [21] & 50 & 92 & $4.0 / 7.1$ & $-44 \%$ & $6 \times 10^{-13}$ & 56.3 \\
\hline Petrus et al. 1998 [22] & 101 & 89 & $3.8 / 5.1$ & $-26 \%$ & 0.0033 & 11.4 \\
\hline Turner at al. A 2000 [23] & 139 & 80 & $6.0 / 5.5$ & & 0.5 & 1.4 \\
\hline Mossad et al.1996 [24] & 99 & 80 & $4.4 / 7.6$ & $-42 \%$ & 0.0005 & 15.2 \\
\hline Prasad et al. 2000 [25] & 48 & 80 & $4.5 / 8.1$ & $-44 \%$ & $2 \times 10^{-9}$ & 40.0 \\
\hline Turner et al. B 2000 [23] & 139 & 69 & $5.5 / 5.5$ & & 0.5 & 1.4 \\
\hline Douglas et al. 1987 [26] & $58^{\circ}$ & 64 & $12.1 / 7.7$ & & 0.96 & 0.1 \\
\hline Macknin et al. 1998 [27] & 247 & 45 & $9.0 / 9.0$ & & 0.5 & 1.4 \\
\hline Weismann et al. 1990 [28] & 130 & 45 & $7 / 6$ & & 0.5 & 1.4 \\
\hline Turner et al. C 2000 [23] & 143 & 30 & $6.0 / 5.5$ & & 0.5 & 1.4 \\
\hline
\end{tabular}

Source: (Jackson et al., 2020)

Literature review on the validities of zinc tablets on the symptoms of respiratory illness computed the impacts of zinc supplements on the duration of the respiratory sickness on the absolute scale, that is, the variation in days in the length of the common cold. Table 1 indicates a notable disparityin the duration of common cold symptoms in the palliative categories, from 5.19.0 days and 10.8 days (Adhikari, \& Das, 2016 457). While afraction of this dissimilarity is triggered by random variation, as well asa real difference in the gravity of illness in various patients and distinct result descriptions. Thus, this review calculated the comparative effects of zinc on the typical duration of common cold symptoms in percentages. The effects partially adjust for the differences between the patient group and result descriptions. In a previous review, computations of the relative effects rather than the absolute impact established a shred of more robust proof that vitamin $\mathrm{C}$ supplements reduces the time of the common cold symptoms (Johnstone et al., 2015). Thus, the comparative impact is utilized in the Cochrane study on vitamin $\mathrm{C}$ and the symptoms of respiratory disease. An assessment of the findings of zinc trials, using $\mathrm{P}$-values together with the Fisher method and combining the survey outcomes were assessed through the RevMan program.

Table 2. The Effect of Zinc Lozenges on the Duration of the Common Cold: Combining the P-Values of the Placebo-Controlled Trials

\begin{tabular}{|l|c|c|c|c|}
\hline \multicolumn{1}{|c|}{ Trials Being Combined } & No. of Trials & $\chi^{2}$ & df & $P$ \\
\hline \hline All trials & 13 & 154.0 & 26 & $10^{-19}$ \\
\hline Low $\mathrm{Zn}$ dose $(<75 \mathrm{mg} /$ day $)$ & 5 & 5.7 & 10 & 0.8 \\
\hline High $\mathrm{Zn}$ dose $(>75 \mathrm{mg} /$ day $)$ & 8 & 148.3 & 16 & $10^{-22}$ \\
\hline Zn-acetate $[21,22,25]$ & 3 & 107.7 & 6 & $10^{-20}$ \\
\hline not $\mathrm{Zn}$-acetate $[7,19,20,23,24]$ & 5 & 40.6 & 10 & $10^{-5}$ \\
\hline
\end{tabular}

The P-values of the individual trials are combined by using the Fisher method (see the Methods section). The combined $\chi^{2}$ value is calculated from the $-2 \times \ln (\mathrm{P})$ values on the right side of Table 1 . The combined P-values are separately calculated for low dose and high dose trials. Finally, the high dose trials are divided to those which used zinc acetate and to those which used zinc salts other than acetate.

Source: (Jackson et al., 2020)

\section{Results}

From this demonstration, 13 palliative-controlled relationships have evaluated the medicinal impacts of zinc tablets on the duration of common cold symptoms. All 13relations were doubleblind; hence the trials utilized allocation, suppression. According to Adhikari \& Das (2016, 457), experiments were randomized. In Table 1, the tests are arranged by the total everyday amount of rudimentary $\mathrm{Zn}$ in $\mathrm{mg}$ from the tablets. 
The seven varied results found statistically significant benefits from zinc tablets. From the outcomes in Table 1, a considerable ratio of the disparity in the consequences might be elucidated by the everyday zinc prescription. Seven out of the eight relationships that used more than $75 \mathrm{mg}$ of zinc per day established a statistically essential benefit. In contrast, the benefits in the Jackson et al. (2020) trial limited to the symptom's acuteness at the late stage of the common cold. The P-values of each test pooled by the Fisher method in Table 2 offers definite proof that zinc tablets and placebo categories are distinct in all the trials. However, zinc lozenges' notable impact is noted differently in three high-dosage tests where $\mathrm{Zn}$ acetate was utilized and in five high-dose tests that utilized zinc salts instead of acetate (Adhikari, \& Das,2016 458). Combining the three high-dose zinc acetate tests provide a decline in the duration of colds, and there is "heterogeneity" observed between the tests.

\section{Discussion}

In the trials involving daily dosage below $75 \mathrm{mg}$, there was no validities of zinc supplements observed. However, for the trials involving more than $75 \mathrm{mg}$ of zinc dosage, there were apparent benefits (Johnstone et al., 2015). Therefore,from this study, zinc cleared symptoms such as headache, hoarseness, sore throat, and nasal congestion. However, in the trials, zinc supplements brought some side effects such as constipation and bad taste reactions (Turner, 2016 5). Such reported side effects, exceptionally lousy taste, might have been fostered by some tablet conformation, and cannot mirror the impacts of $\mathrm{Zn}^{2+}$.

In a contemporary experiment on zinc acetate, there were insignificant variations between the zinc and placebo classesin the situations of unpleasant impacts while the dosage was $92 \mathrm{mg}$ (Adhikari, \& Das, 2016 458). A Cochrane review established that zinc lozenges inhibit the replication of the virus that triggers common cold and shortens the duration of the disease when taken in a $75 \mathrm{mg}$ /day dosage, within 24 hours of symptom onset (Suara, \& Crowe, 2016 785). Thus, from this analysis, we can establish that zinc may inhibit SARS-CoV-2. According to cell cultures, zinc concentrations and the supplement of Pyrithione for incentive of the cellular import of zinc ions leads to inhibition of the replication of different RNA viruses (Velthuis et al., 2016), such as coronavirus. Also, zinc deters the RNAproducing events of Nidoviruses, like SARS-CoV (Suara, \& Crowe, 2016 785), in vitro, which is achieved by altering RdRp functions throughout the elongation stage of RNA production, by absolutely impacting template combining (Velthuis et al., 2016). In this light, just like other coronaviruses, COVID-19 causing virus, SARSCoV-2 also comes under the category of Nidoviruses (Skalny et al., 2020 20).

This review demonstrated that orally dispensed zinc supplements reduced the duration of cold symptoms. While there was no indication that people taking zinc tablets were symptomatic at 14 days, there was no variation between categories in the acuteness of the symptoms. Nonetheless, zinc has been established to shorten the duration of cold virus symptoms, where SARS-CoV is also a member of the family of viruses (Turner, 20165 ). The effect was highly attenuated and statistically significant in children. Symptoms of the disease were generally less severe in children and teens compared with adults. This is because serum cytokines were at higher levels in children than adults, also levels of two immune system molecules — interleukin 17A (IL-17A), which helps mobilize immune system response during early infection, and interferon gamma (INF-g), which combats viral replication - were strongly linked to the age of the patients. The younger the patient, the higher the levels of IL-17A and INF-g. Hence, the findings from this survey suggest that there is a potential dose-reliant impact linked with zinc ion, and it is coherent with the magnitude of medical response. This study differs in several instances from the Cochrane survey (Rahman \& Idid, 2020, 3). Although this report was limited to 
zinc supplements, Cochrane research pools zinc lozenges with normal zinc tablet tests. Nonetheless, the advantages of zinc tablets are likely characterized by low early zinc ingestion of the patients. Consequently, the impacts of zinc tablets might be brought about by local impacts of the oral cleft (Rahman \& Idid, 2020, 3). As Findings from animal experiments suggest a link between poor maternal zinc status and increased risk of oral clefts in offspring; however, there are few human studies on this issue. As higher plasma zinc concentrations were associated with a lower risk for oral clefts in the children. However, the Cochrane review established that zinc supplementation can shorten the average duration of coronavirus symptoms when taken within 24 hours. The symptoms appear at a dosage above 75 mg per day.

\section{Conclusion}

In a nutshell, the systematic review has proposed that zinc lozenges can reduce the duration of symptoms and severity of disease of the coronavirus when consumed within a day on the onset of the symptoms. SARS-CoV-2 is transmitted from one person to another, and the symptomatic people are the most significant carriers of the virus. Like other respiratory illnesses, the spread of SARS-CoV-2 is perceived to happen via respiratory droplets from sneezing and coughing. Currently, treatment mechanisms to manage COVID-19 are only supportive care and decreasing community spread. Nonetheless, this systematic review indicates that zinc supplementation can suppress coronavirus symptoms by inhibiting the replication of the respiratory virus. Thus, zinc has several purposes on the defense structure, and its insufficiency augments the susceptibility to diseases. The research findings indicate that zinc tablets can reduce the danger of acute respiratory illnesses, such as COVID-19. In cell culture, Pyrithione fosters zinc ions uptake within a short time and inhibits ribonucleic acid virus replication through a strategy that has been reviewed for picornaviruses. There were apparent benefits from the trials involving more than $75 \mathrm{mg}$ of zinc dosage from the results reviewed above. As such, the intake of zinc lozenges cleared the coronavirus's (common cold) symptoms such as headache, hoarseness, sore throat, and nasal congestion. Similarly, a Cochrane review established that zinc lozenges inhibit the replication of the virus that triggers respiratory illnesses and shortens the duration of the disease when taken in a $75 \mathrm{mg} /$ day dosage, within 24 hours of initial symptoms. Zinc hinders the RNAproducing events of Nidoviruses, like SARS-CoV, in vitro, which is achieved by altering $\mathrm{RdRp}$ functions during the elongation stage of RNA production, by explicitly impacting prototype requisite. Therefore, from this analysis, we can establish that zinc can inhibit SARS-CoV-2. However, the intervention is likely to face challenges since according to $\mathrm{WHO}$ and $\mathrm{CDC}$, the majority of COVID-19 patients are asymptomatic $(80 \%)$. It is therefore difficult to determine the most appropriate time to initialize consumption of zinc lozenges. However, administration of zinc lozenges to all individuals could have protective effects to potentially reduce COVID-19symptoms, as well as the spread.

\section{References}

1. Adhikari, D. D., \& Das, S. (2016). Role of Zinc Supplementation in the Outcome of Repeated Acute Respiratory Infections in Indian Children: A Randomized DoubleBlind Placebo-Controlled Clinical Trial. Research Journal of Pharmacy and Technology, 9(4), 457-458.

2. Cascella, M., Rajnik, M., Cuomo, A., Dulebohn, S. C., \& Di Napoli, R. (2020). Features, evaluation and treatment coronavirus (COVID-19). In Statpearls [internet]. StatPearls Publishing.

3. Jackson, J. L., Lesho, E., \& Peterson, C. (2020). Zinc and the common cold: a meta-analysis revisited-the Journal of Nutrition, 130(5), 1512S-1515S. 
4. Johnstone, J., Roth, D. E., Guyatt, G., \& Loeb, M. (2015). Zinc for the treatment of the common cold: a systematic review and meta-analysis of randomized controlled trials. Cmaj, 184(10), E551-E561.

5. Kumar, A., Kubota, Y., Chernov, M., \&Kasuya, H. (2020). Potential Role of Zinc Supplementation in Prophylaxis and Treatment of COVID-19. Medical Hypotheses, 109848.

6. Mousavizadeh, L., \&Ghasemi, S. (2020). Genotype and phenotype of COVID-19: Their roles in pathogenesis. Journal of Microbiology, Immunology and Infection.

7. Nair, B. T., Bhunia, R., \& Sharma, K. K. (2017). Role of zinc supplementation in acute respiratory tract infections in children aged 2 to 60 months. International Journal of Contemporary Pediatrics, 4(5), 1758-62.

8. Rahman, M. T., \& Idid, S. Z. (2020). Can $\mathrm{Zn}$ Be a Critical Element in COVID-19 Treatment? Biological Trace Element Research, 1-9.

9. Skalny, A. V., Rink, L., Ajsuvakova, O. P., Aschner, M., Gritsenko, V. A., Alekseenko, S. I., ... \&Tsatsakis, A. (2020). Zinc and respiratory tract infections: Perspectives for COVID-19. International Journal of Molecular Medicine, 46(1), 17-26.

10. Suara, R. O., \& Crowe, J. E. (2016). Effect of zinc salts on respiratory syncytial virus replication. Antimicrobial agents and chemotherapy, 48(3), 783-790.

11. Turner, R. B. (2016). The treatment of rhinovirus infections: progress and potential. Antiviral Research, 49(1), 1-14.

12. Velthuis, A. J., Worm, S. H., Sims. (2016). $\mathrm{Zn} 2+$ inhibits coronavirus, and arterivirus RNA polymerase activity in vitro and zinc ionophores block the replication of these viruses in cell culture. PLoS pathogens, 6(11), e1001176.
13. Worldometers.info. 2020. Coronavirus Update (Live): 13,855,229 Cases and 589,947 Deaths From COVID-19 Virus Pandemic - Worldometer. [online] Available at: <https://www.worldometers.info/coronavir us/?utm_campaign=homeAdvegas1?>

[Accessed 16 July 2020]

14. teVelthuis, A., van den Worm, S., Sims, A., Baric, R., Snijder, E. and van Hemert, M., 2020. Zn2+ Inhibits Coronavirus And Arterivirus RNA Polymerase Activity In Vitro And Zinc Ionophores Block The Replication Of These Viruses In Cell Culture. 